\title{
SOME EXTENSIONS OF THE MEHLER FORMULA ${ }^{1}$
}

\author{
H. M. SRIVASTAVA AND J. P. SINGHAL
}

Abstract. By using certain operational techniques, the authors prove an elegant unification of several extensions of the well-known Mehler formula for Hermite polynomials, given recently by L. Carlitz ([1], [2]). It is also shown how rapidly a number of Carlitz's formulas would follow from these considerations. The last section discusses a generalization involving the product of several Hermite polynomials of different arguments.

1. Introduction. In the theory of Hermite polynomials $\left\{H_{n}(z)\right\}$ defined by

$$
\sum_{n=0}^{\infty} H_{n}(z) \frac{t^{n}}{n !}=\exp \left(2 z t-t^{2}\right)
$$

the bilinear generating function

$$
\sum_{n=0}^{\infty} H_{n}(x) H_{n}(y) \frac{t^{n}}{n !}=\left(1-4 t^{2}\right)^{-1 / 2} \exp \left\{\frac{4 x y t-4\left(x^{2}+y^{2}\right) t^{2}}{1-4 t^{2}}\right\}
$$

is well known as Mehler's formula [3, p. 198]. Recently, Carlitz ([1], [2]) has proved a number of extensions of (1.2). In particular, we recall here his elegant formula

where

$$
\begin{aligned}
\sum_{m, n, p=0}^{\infty} H_{n+p}(x) H_{p+m}(y) H_{m+n}(z) \frac{u^{m}}{m !} \frac{v^{n}}{n !} \frac{w^{p}}{p !} \\
=\Delta^{-1 / 2} \exp \left\{\sum x^{2}-\frac{1}{\Delta}\left(\sum x^{2}-4 \sum u^{2} x^{2}\right.\right. \\
\left.\left.-4 \sum w x y+8 \sum u v x y\right)\right\}
\end{aligned}
$$

$$
\Delta=1-4 u^{2}-4 v^{2}-4 w^{2}+16 u v w,
$$

Presented to the Society, November 30, 1970; received by the editors December 1, 1970.

AMS 1970 subject classifications. Primary 33A65, 33A45; Secondary 42A52.

Key words and phrases. Mehler's formula, operational techniques, Hermite polynomials, bilinear generating function, Rodrigues' formula, Glaisher's formula.

${ }^{1}$ This work was supported in part by the National Research Council of Canada under Grant A7353.

(c) American Mathematical Society 1972 
and $\sum x^{2}, \sum u^{2} x^{2}, \sum w x y, \sum u v x y$ are symmetric functions in the indicated variables.

The two proofs of (1.3), given by Carlitz [2], seem to be long and involved. In the present note we first give a simple and direct proof of (1.3) by using certain operational techniques. We then proceed to derive the following result which generalizes (1.3) and a number of other extensions of (1.2) proved by Carlitz ([1, p. 45, equation (8)]; [2, pp. 117-118, equations (1.2), (1.4), (1.6), (1.7)]).

$$
\begin{aligned}
\sum_{m, n, p=0}^{\infty} & H_{n+p+r}(x) H_{p+m+s}(y) H_{m+n}(z) \frac{u^{m}}{m !} \frac{v^{n}}{n !} \frac{w^{p}}{p !} \\
= & \Delta^{-1 / 2}\left(1-4 u^{2}\right)^{r / 2}\left(1-4 v^{2}\right)^{s / 2} \\
& \cdot \exp \left\{\sum x^{2}-\frac{1}{\Delta}\left(\sum x^{2}-4 \sum u^{2} x^{2}-4 \sum w x y+8 \sum u v x y\right)\right\} \\
& \cdot \cdot \sum_{k=0}^{\min (r, s)} 2^{2 k} k !\left(\begin{array}{l}
r \\
k
\end{array}\right)\left(\begin{array}{l}
s \\
k
\end{array}\right)\left(\frac{w-2 u v}{\left(\left(1-4 u^{2}\right)\left(1-4 v^{2}\right)\right)^{1 / 2}}\right)^{k} \\
& \cdot H_{r-k}\left(\frac{(x-2 v z)\left(1-4 u^{2}\right)-2(y-2 u z)(w-2 u v)}{\left(\Delta\left(1-4 u^{2}\right)\right)^{1 / 2}}\right) \\
& \cdot H_{s-k}\left(\frac{(y-2 u z)\left(1-4 v^{2}\right)-2(x-2 v z)(w-2 u v)}{\left(\Delta\left(1-4 v^{2}\right)\right)^{1 / 2}}\right),
\end{aligned}
$$

where $\Delta$ is given by (1.4).

In our analysis we shall make use of a number of known results which we mention here for ready reference.

$$
D_{x}^{r} H_{n}(x)=2^{r} r !\left(\begin{array}{l}
n \\
r
\end{array}\right) H_{n-r}(x),
$$

where, as usual, $D_{z} \equiv d / d z$.

$$
\begin{gathered}
\exp \left(t D_{x}\right) f(x)=f(x+t) . \\
H_{n}(a x)=(-1 / a)^{n} \exp \left(a^{2} x^{2}\right) D_{x}^{n} \exp \left(-a^{2} x^{2}\right),
\end{gathered}
$$

which follows at once from Rodrigues' formula for the Hermite polynomial.

$$
\exp \left(t D_{x}^{2}\right)\left\{\exp \left(-x^{2}\right)\right\}=(1+4 t)^{-1 / 2} \exp \left\{\frac{-x^{2}}{1+4 t}\right\},
$$

which is Glaisher's operational formula.

$$
\begin{aligned}
\exp \left(t D_{x} D_{y}\right) & \left\{\exp \left(-a^{2} x^{2}-b^{2} y^{2}\right)\right\} \\
& =\left(1-4 a^{2} b^{2} t^{2}\right)^{-1 / 2} \exp \left\{-a^{2} x^{2}-\frac{\left(b y-2 a^{2} b x t\right)^{2}}{1-4 a^{2} b^{2} t^{2}}\right\},
\end{aligned}
$$

which was derived earlier by Singhal [4]. 
2. Proof of (1.3). Denoting the left-hand side of (1.3) by $\Omega$, if we make use of (1.8) and (1.1), we get

$$
\begin{aligned}
\Omega= & \sum_{m, n=0}^{\infty} H_{m+n}(z) \frac{u^{m}}{m !} \frac{v^{n}}{n !} \sum_{p=0}^{\infty} H_{n+p}(x) H_{p+m}(y) \frac{w^{p}}{p !} \\
= & \sum_{m, n=0}^{\infty} H_{m+n}(z) \frac{u^{m}}{m !} \frac{v^{n}}{n !} \\
& \cdot \sum_{p=0}^{\infty} \exp \left(x^{2}+y^{2}\right)\left(-D_{x}\right)^{n+p}\left(-D_{y}\right)^{p+m} \exp \left(-x^{2}-y^{2}\right) \\
= & \exp \left(x^{2}+y^{2}\right) \\
& \cdot \sum_{m, n=0}^{\infty} H_{m+n}(z) \frac{\left(-u D_{y}\right)^{m}}{m !} \frac{\left(-v D_{x}\right)^{n}}{n !} \exp \left(w D_{x} D_{y}\right) \exp \left(-x^{2}-y^{2}\right) \\
= & \exp \left(x^{2}+y^{2}\right) \\
& \cdot \sum_{k=0}^{\infty} H_{k}(z) \frac{\left(-u D_{y}-v D_{x}\right)^{k}}{k !} \exp \left(w D_{x} D_{y}\right) \exp \left(-x^{2}-y^{2}\right) \\
= & \exp \left(x^{2}+y^{2}\right) \\
& \cdot \exp \left\{-2 u z D_{y}-2 v z D_{x}+(w-2 u v) D_{x} D_{y}-u^{2} D_{y}^{2}-v^{2} D_{x}^{2}\right\} \\
& \left.\cdot \cdot \exp \left(-x^{2}-y^{2}\right)\right\} \\
= & \left(1-4 u^{2}\right)^{-1 / 2}\left(1-4 v^{2}\right)^{-1 / 2} \exp \left(x^{2}+y^{2}\right) \\
& \cdot \exp \left\{-2 u z D_{y}-2 v z D_{x}+(w-2 u v) D_{x} D_{y}\right\} \\
& \cdot \exp \left\{-\frac{x^{2}}{1-4 v^{2}}-\frac{y^{2}}{1-4 u^{2}}\right\}
\end{aligned}
$$

by virtue of Glaisher's formula (1.9).

Now we apply formulas (1.10) and (1.7), and we observe that

$$
\begin{aligned}
\Omega= & \Delta^{-1 / 2} \exp \left(x^{2}+y^{2}\right) \exp \left\{-2 u z D_{y}-2 v z D_{x}\right\} \\
& \cdot \exp \left\{\frac{-x^{2}\left(1-4 u^{2}\right)-y^{2}\left(1-4 v^{2}\right)+4 x y(w-2 u v)}{\Delta}\right\} \\
= & \Delta^{-1 / 2} \exp \left(x^{2}+y^{2}\right) \\
& \cdot \exp \left\{-\frac{1}{\Delta}\left((x-2 v z)^{2}\left(1-4 u^{2}\right)-(y-2 u z)^{2}\left(1-4 v^{2}\right)\right.\right. \\
& +4(x-2 v z)(y-2 u z)(w-2 u v))\} \\
= & \Delta^{-1 / 2} \exp \left\{\sum x^{2}-\frac{1}{\Delta}\left(\sum x^{2}-4 \sum u^{2} x^{2}-4 \sum w x y+8 \sum u v x y\right)\right\},
\end{aligned}
$$

which completes the proof of Carlitz's formula (1.3). 
3. Proof of (1.5). Following an analysis similar to the one used in the preceding section, it is readily seen that

$$
\begin{aligned}
& \sum_{m, n, p=0}^{\infty} H_{n+p+r}(x) H_{p+m+s}(y) H_{m+n}(z) \frac{u^{m}}{m !} \frac{v^{n}}{n !} \frac{w^{p}}{p !} \\
& =\Delta^{-1 / 2} \exp \left(x^{2}+y^{2}\right) \exp \left(-2 u z D_{y}-2 v z D_{x}\right) \\
& \cdot\left(-D_{x}\right)^{r}\left(-D_{y}\right)^{s} \exp \left\{-\frac{x^{2}}{1-4 v^{2}}-\frac{\left(y\left(1-4 v^{2}\right)-2 x(w-u v)\right)^{2}}{\Delta\left(1-4 v^{2}\right)}\right\} \\
& =\Delta^{-(s+1) / 2}\left(1-4 v^{2}\right)^{s / 2} \exp \left(x^{2}+y^{2}\right) \exp \left\{-2 u z D_{y}-2 v z D_{x}\right\} \\
& \cdot\left(-D_{x}\right)^{r} \exp \left\{-\frac{x^{2}}{1-4 v^{2}}-\frac{\left(y\left(1-4 v^{2}\right)-2 x(w-2 u v)\right)^{2}}{\Delta\left(1-4 v^{2}\right)}\right\} \\
& \cdot H_{s}\left(\frac{y\left(1-4 v^{2}\right)-2 x(w-2 u v)}{\left(\Delta\left(1-4 v^{2}\right)\right)^{1 / 2}}\right) \\
& =\Delta^{-(s+1) / 2}\left(1-4 v^{2}\right)^{s / 2} \exp \left(x^{2}+y^{2}\right) \exp \left\{-2 u z D_{y}-2 v z D_{x}\right\} \\
& \cdot \sum_{k=0}^{r}(-1)^{r}\left(\begin{array}{l}
r \\
k
\end{array}\right) D_{x}^{r-k} \\
& \cdot \exp \left\{-\frac{\left(x\left(1-4 u^{2}\right)-2 y(w-2 u v)\right)^{2}}{\Delta\left(1-4 u^{2}\right)}-\frac{y^{2}}{1-4 u^{2}}\right\} \\
& \text { - } D_{x}^{k} H_{s}\left(\frac{y\left(1-4 v^{2}\right)-2 x(w-2 u v)}{\left(\Delta\left(1-4 v^{2}\right)\right)^{1 / 2}}\right) \\
& =\Delta^{-(r+s+1) / 2}\left(1-4 u^{2}\right)^{r / 2}\left(1-4 v^{2}\right)^{s / 2} \exp \left(x^{2}+y^{2}\right) \\
& \text { - } \exp \left(2 u z D_{y}-2 v z D_{x}\right) \\
& \cdot \exp \left\{\frac{-x^{2}\left(1-4 u^{2}\right)-y^{2}\left(1-4 v^{2}\right)+4 x y(w-2 u v)}{\Delta}\right\} \\
& \cdot \sum_{k=0}^{\min (r, s)} 2^{2 k} k !\left(\begin{array}{l}
r \\
k
\end{array}\right)\left(\begin{array}{l}
s \\
k
\end{array}\right)\left(\frac{w-2 u v}{\left(\left(1-4 u^{2}\right)\left(1-4 v^{2}\right)\right)^{1 / 2}}\right)^{k} \\
& \cdot H_{r-k}\left(\frac{x\left(1-4 u^{2}\right)-2 y(w-2 u v)}{\left(\Delta\left(1-4 u^{2}\right)\right)^{1 / 2}}\right) \\
& \cdot H_{s-k}\left(\frac{y\left(1-4 v^{2}\right)-2 x(w-2 u v)}{\left(\Delta\left(1-4 v^{2}\right)\right)^{1 / 2}}\right) \text {, }
\end{aligned}
$$

which, in view of (1.7), leads us to the desired result (1.5).

4. Particular cases of (1.5). Evidently, (1.5) provides a generalization of Carlitz's formula (1.3) to which it would reduce when $r=s=0$. 
For $u=v=0$, our formula (1.5) leads us to

$$
\begin{aligned}
\sum_{n=0}^{\infty} H_{n+r}(x) H_{n+s}(y) \frac{t^{n}}{n !} & \\
= & \left(1-4 t^{2}\right)^{-(r+s+1) / 2} \exp \left\{\frac{4 x y t-4\left(x^{2}+y^{2}\right) t^{2}}{1-4 t^{2}}\right\} \\
& \quad \cdot \sum_{k=0}^{\min (r, s)} 2^{2 k} k !\left(\begin{array}{l}
r \\
k
\end{array}\right)\left(\begin{array}{l}
s \\
k
\end{array}\right) t^{k} H_{r-k}\left(\frac{x-2 y t}{\left(1-4 t^{2}\right)^{1 / 2}}\right) H_{s-k}\left(\frac{y-2 x t}{\left(1-4 t^{2}\right)^{1 / 2}}\right),
\end{aligned}
$$

which was proved by Carlitz [1] in a different way (see also [4]).

Yet another special case of (1.5) would seem to occur when $u$ or $v$ equals zero. Indeed we thus get the formula

$$
\begin{aligned}
& \sum_{m, n=0}^{\infty} H_{m+n+r}(x) H_{m}(y) H_{n+s}(z) \frac{u^{m}}{m !} \frac{v^{n}}{n !} \\
&=\left(1-4 u^{2}-4 v^{2}\right)^{-(r+s+1) / 2}\left(1-4 u^{2}\right)^{s / 2} \\
& \cdot \exp \left\{\frac{-4 x^{2}\left(u^{2}+v^{2}\right)+4 x(u y+v z)-4(u y+v z)^{2}}{1-4 u^{2}-4 v^{2}}\right\} \\
& \cdot \sum_{k=0}^{\min (r, s)} 2^{2 k} k !\left(\begin{array}{l}
r \\
k
\end{array}\right)\left(\begin{array}{l}
s \\
k
\end{array}\right)\left(\frac{v}{\left(1-4 u^{2}\right)^{1 / 2}}\right)^{k} H_{r-k}\left(\frac{x-2 u y-2 v z}{\left(1-4 u^{2}-4 v^{2}\right)^{1 / 2}}\right) \\
& \cdot H_{s-k}\left(\frac{z-4 u^{2} z-2 v x+4 u v y}{\left(\left(1-4 u^{2}\right)\left(1-4 u^{2}-4 v^{2}\right)\right)^{1 / 2}}\right),
\end{aligned}
$$

which is believed to be new. For $s=0$, formula (4.2) would further reduce to the elegant result

$$
\begin{aligned}
\sum_{m, n=0}^{\infty} H_{m+n+r} & (x) H_{m}(y) H_{n}(z) \frac{u^{m}}{m !} \frac{v^{n}}{n !} \\
= & \left(1-4 u^{2}-4 v^{2}\right)^{-(r+1) / 2} \\
& \cdot \exp \left\{\frac{-4 x^{2}\left(u^{2}+v^{2}\right)+4 x(u y+v z)-4(u y+v z)^{2}}{1-4 u^{2}-4 v^{2}}\right\} \\
& \cdot H_{r}\left(\frac{x-2 u y-2 v z}{\left(1-4 u^{2}-4 v^{2}\right)^{1 / 2}}\right),
\end{aligned}
$$

which provides a generalization of Carlitz's formula (see $[2$, p. 117, equation (1.2)])

$$
\begin{aligned}
& \sum_{m, n=0}^{\infty} H_{m+n}(x) H_{m}(y) H_{n}(z) \frac{u^{m}}{m !} \frac{v^{n}}{n !}=\left(1-4 u^{2}-4 v^{2}\right)^{-1 / 2} \\
& \cdot \exp \left\{\frac{-4 x^{2}\left(u^{2}+v^{2}\right)+4 x(u y+v z)-4(u y+v z)^{2}}{1-4 u^{2}-4 v^{2}}\right\}
\end{aligned}
$$


which follows at once from (4.3) when $r=0$. Lastly, on setting $w=0$, (1.5) would give us

$$
\begin{aligned}
\sum_{m, n=0}^{\infty} H_{m+n}(x) & H_{m+s}(y) H_{n+r}(z) \frac{u^{m}}{m !} \frac{v^{n}}{n !} \\
= & \left(1-4 u^{2}-4 v^{2}\right)^{-(r+s+1) / 2}\left(1-4 u^{2}\right)^{r / 2}\left(1-4 v^{2}\right)^{s / 2} \\
& \cdot \exp \left\{\frac{-4 x^{2}\left(u^{2}+v^{2}\right)+4 x(u y+v z)-4(u y+v z)^{2}}{1-4 u^{2}-4 v^{2}}\right\} \\
& \cdot \sum_{k=0}^{\min (r, s)} 2^{2 k} k !\left(\begin{array}{l}
r \\
k
\end{array}\right)\left(\begin{array}{l}
s \\
k
\end{array}\right)\left(\frac{-2 u v}{\left(\left(1-4 u^{2}\right)\left(1-4 v^{2}\right)\right)^{1 / 2}}\right)^{k} \\
& \cdot H_{r-k}\left(\frac{z\left(1-4 u^{2}\right)-2 v(x-2 u y)}{\left(\left(1-4 u^{2}\right)\left(1-4 u^{2}-4 v^{2}\right)\right)^{1 / 2}}\right) \\
\cdot & H_{s-k}\left(\frac{y\left(1-4 v^{2}\right)-2 u(x-2 v z)}{\left(\left(1-4 v^{2}\right)\left(1-4 u^{2}-4 v^{2}\right)\right)^{1 / 2}}\right) .
\end{aligned}
$$

Note that formula (4.5), with a different right-hand side, was also proved by Carlitz ([2, p. 118, equation (1.7)]).

It may be of interest to conclude with the remark that formulas (4.2) and (4.3) admit themselves of an elegant generalization in the form

$$
\begin{gathered}
\sum_{m, n_{1}, \cdots, n_{k}=0}^{\infty} H_{m+n_{1}+\cdots+n_{k}+r}(x) H_{m+s}(y) H_{n_{1}}\left(z_{1}\right) \cdots H_{n_{k}}\left(z_{k}\right) \frac{u^{m}}{m !} \frac{v_{1}^{n_{1}}}{n_{1} !} \cdots \frac{v_{k}^{n_{k}}}{n_{k} !} \\
=\left(1-4 u^{2}-4 \sum v_{i}^{2}\right)^{-(r+s+1) / 2}\left(1-4 \sum v_{i}^{2}\right)^{s / 2} \\
\cdot \exp \left\{x^{2}-\frac{\left(x-2 u y-2 \sum v_{i} z_{i}\right)^{2}}{1-4 u^{2}-4 \sum v_{i}^{2}}\right\} \\
\cdot \sum_{k=0}^{\min (r, s)} 2^{2 k} k !\left(\begin{array}{l}
r \\
k
\end{array}\right)\left(\begin{array}{l}
s \\
k
\end{array}\right)\left(\frac{u}{\left(1-4 \sum v_{i}^{2}\right)^{1 / 2}}\right)^{k} \\
\cdot H_{r-k}\left(\frac{x-2 u y-2 \sum v_{i} z_{i}}{\left(1-4 u^{2}-4 \sum v_{i}^{2}\right)^{1 / 2}}\right) \\
\cdot H_{s-k}\left(\frac{y\left(1-4 \sum v_{i}^{2}\right)-2 u\left(x-2 \sum v_{i} z_{i}\right)}{\left\{\left(1-4 u^{2}-4 \sum v_{i}^{2}\right)\left(1-4 \sum v_{i}^{2}\right)\right\}^{1 / 2}}\right),
\end{gathered}
$$

where the range of each $i$ summation on the right-hand side is from $i=1$ to $i=k, k=1,2,3 \cdots$.

The proof of (4.6) would make use of the known formulas (1.6), (1.8), and (1.9) in a manner already illustrated in $\$ \S 2$ and 3.

Incidentally, (4.6) corresponds to Carlitz's formula (2.3) in [2, p. 120] when $r=s=u=0$. 


\section{REFERENCES}

1. L. Carlitz, An extension of Mehler's formula, Boll. Un. Mat. Ital. (4) 3 (1970), 43-46.

2. - Some extensions of the Mehler formula, Collect. Math. 21 (1970), 117-130.

3. E. D. Rainville, Special functions, 3rd ed., Macmillan, New York, 1965.

4. J. P. Singhal, Certain extensions of the Mehler formula, Canad. Math. Bull. (to appear); see also: Notices Amer. Math. Soc. 17 (1970), 956. Abstract \#70T-B215.

Department of Mathematics, University of Victoria, Victoria, British Columbia, Canada 